\title{
Bleach Solution Requirement for Hatching of Daphnia magna Resting Eggs
}

\author{
Catur Retnaningdyah ${ }^{1 *}$, Dieter Ebert ${ }^{2}$
}

\author{
${ }^{1}$ Biology Study Program, Faculty of Mathematics and Natural Sciences, Brawijaya University, Malang, Indonesia \\ ${ }^{2}$ Department of Evolutionary Biology, Zoological Institute, University of Basel, Basel, Switzerland
}

\section{ABSTRACT}

Daphnia (water fleas) belong to the zooplankton group called Cladocerans have sexual reproduction when conditions less favorable that produce diapausing eggs are enclosed in the ephippium. Hatching ephippial eggs in the laboratory is important in ecological, toxicology, genetical, and evolutionary studies. This study aims to improve the current methods of egg hatching from ephippium. Each of 50 ephippium were treated together by placing them in a glass jar and adding $50 \mathrm{~mL}$ bleach solution (sodium hypochlorite). Concentrations of sodium hypochlorite used in this experiment were $0 \%, 0.5 \%, 1 \%, 2 \%, 4 \%$ and $8 \%$. These concentration treatments were crossed with the following exposure times $(1,2,4,8,16$, and 32 minutes). Culturing was done in $80 \mathrm{~mL}$ of artificial Daphnia medium, incubated in constant light and temperatures $20^{\circ} \mathrm{C}$ for 25 days. There were two repetitions in this experiment that were run at the same time. Result of this experiment showed that pretreatment with $0.5-8 \%$ bleach solution significantly increases the yield of total hatch rate of Daphnia magna resting eggs by about $21 \%$ over unbleached control. However, there was no significant difference among the bleach treatments. Concentration of bleach solution $0.5 \%, 1 \%$ and $4 \%$ significantly accelerated the time period until the first hatching (first day hatching). Difference of exposure time (1 - 32 minutes) at each concentration treatments were not influence the yield of total hatch and the time period until first hatching.

Keywords: Bleach solution, D. magna resting eggs, hatching

\section{INTRODUCTION}

Daphnia belong to the zooplankton group referred to as Cladocerans (Crustacea), often called water fleas. Daphnia magna is the largest European species of the genus, reaching an adult size of up to $5 \mathrm{~mm}$ [1]. Reproduction of Daphnia is characterized by alternating asexual (parthenogenic) and sexual stages [2,3].

Parthenogenesis, or asexual reproduction, is the mode which Cladocerans utilize for most of the year. The Daphnia multiply by parthenogenesis when favorable conditions such as their food is abundant. The Cladocerans molt to increase in size throughout their life. Following each molt, the mature females have between 0 and 50 eggs in their brood chamber. These eggs develop without fertilization into immature females. After the next molting stage, the juveniles are released. These juveniles begin to feed and mature at a

\section{${ }^{*}$ Corresponding author:}

Catur Retnaningdyah

Biology Department, Faculty of Mathematics and Natural

Sciences, Brawijaya University

Jalan Veteran, Malang, Indonesia 65145

E-mail: catur@ub.ac.id rapid pace. Parthenogenesis allows for quick population growth, assuming favorable conditions [4].

Later in the season, when conditions for parthenogenesis become less favorable due to overcrowding, accumulation of metabolic wastes and when food availability and/or temperature begins to decrease, some of the eggs that were produced parthenogenically by the females, hatch into males [4]. The production of males and sexual females is triggered by the environment $[5,6]$. Males are genetically identical to their clonal sisters [7]. Males mate and fertilize haploid diapausing eggs produced by females entering the sexual phase of the reproductive cycle $[8,9]$. The fertilized eggs are kept in the brood chamber of the female. Diapausing eggs are enclosed in the ephippium, a protective structure modified from the carapace that the female moults. When the female next molts, the ephippium

\section{How to cite:}

Retnaningdyah C, Ebert D (2016) Bleach Solution Requirement for Hatching of Daphnia magna Resting Eggs. J. Trop.

Life. Science 6 (3): 136 - 141. 
with its enclosed the resting eggs are released. Resting eggs are important for over-wintering and dispersal. Diapausing eggs survive unfavorable periods, such as when the habitat freezes or dries and act as a dispersal stage, carried by wind, water and birds. They are often blown inshore, where they may overwinter. The eggs hatch in the littoral regions the following spring $[1,4$, $6,10,11]$. Diapause is most commonly considered a way of avoiding harsh abiotic conditions. Besides abiotic extremes such as drying up of the water body, deterioration in food conditions is often indicated as a factor behind diapause induction. Limited food has been found could cause increasing the production of ephippial in D. magna [12].

The termination of diapausing eggs (ephippial) in Daphnia is stimulated by a number of environmental components. The light was known capable to breaks the diapause of ephippial eggs. The diapausing eggs of the Paul Lake strain are clearly activated by light but in a manner which suggests that light is not essential for diapause release under conditions of the experiments. However, activation by light occurred only after the ephippia were soaked in a bleaching solution of sodium hypochlorite $(\mathrm{NaClO})$ which is an effective disinfecting agent and widely used for sterilization of materials. A significantly larger number of young Daphnia hatched from ephippia soaked in a $1 \%$ solution of sodium hypochlorite for 5 minutes and incubated in light, but it did not exceed $17 \%$ [13, 14]. Hatching ephippial eggs in the laboratory is important in ecological, toxicology, genetical and evolutionary studies $[3,15,16]$. Based on the above, the aim of this research is to improve the current methods of egg hatching from ephippia. This paper is a continuation of that work to determine if the bleaching solution is essential for the release of diapause. Besides that, we also determined how the exposure time of sodium hypochlorite influences hatching of $D$. magna resting eggs.

\section{MATERIALS AND METHODS}

Ephippia of $D$. magna for the experiments were gathered from an intermittent carp breeding pond near Munich, Germany. The ephippia are extremely buoyant and are concentrated by the wind in a small area at the margin of the lake. Collection was made in March 1997 when the pond was dry. The material was stored at $4^{\circ} \mathrm{C}$ in complete darkness. This experiment was done in September-November 2004, at Unit Ecology and Evolution, Biology Department, University of Fribourg, Switzerland.
There were two repetitions in this experiment that were run in the same time. The number of ephippia used was 50 per beaker. A sample of ephippia withpond sediment was placed in a net with $200 \mu \mathrm{m}$ mesh size. Ephippia were washed with aquadest. Then we separated the ephippia of $D$. magna from other material. Portions of 50 ephippia were treated together by placing them in a glass jar and adding $50 \mathrm{~mL}$ bleach solution. Concentrations of sodium hypochlorite $(\mathrm{Na}-$ $\mathrm{ClO}$ ) used in this experiment were $0 \%, 0.5 \%, 1 \%, 2 \%$, $4 \%$ and $8 \%$. These concentration treatments were crossed with the following exposure times $(1,2,4,8$, 16, and 32 minutes). After exposing ephippia were washed with $1.5 \mathrm{~L}$ of deinoized water. The subsequent culturing was done in $80 \mathrm{~mL}$ artificial Daphnia medium: ADaM (Ebert, D. Web-guide to Daphnia parasites). Incubation of all replicates was done in constant light and temperatures $20^{\circ} \mathrm{C}$ for 25 days. We used four fluorescent light tubes OSRAM BOLUX Recyclable 36 watt each placed side by side in a distance of $25 \mathrm{~cm}$ over the jars. Hatching yields were determined by counting the number of hatched $D$. magna present at daily intervals. Than these were removed at the time of observation. For the graphical presentation, the counts were converted in a percentage of the eggs hatching. To estimate how many eggs we can expect in 50 ephippia, we opened ephippia. We counted 51 eggs in 75 ephippia, giving an estimate of 0.68 eggs per ephippium or 34 eggs per treatment jars. In this experiment we prepared two samples control with $0 \%$ of bleach solution but used eggs which were taken out of their ephippia.

\section{RESULTS AND DISCUSSION \\ Percentage of egg hatching}

Treatment with $\mathrm{NaClO}$ resulted in a significant increase in percentage of hatch. Hatching rates of ephippial eggs at the treatments of bleach solution when incubated for 25 days varied from $8.8 \%$ to $48.5 \%$. On the other hand the average range of hatched of the control from $4.4 \%$ to $11.8 \%$ (Figure 1). The development of an ephippial eggs about to hatch had observed before [17]. Hatching results from the gradual swelling of subchorionic membrane causing first the equatorial rupture of the chorion and terminating with the instantaneous rupture of the membrane after it has swollen to about twice its original linear dimensions. The swelling is clearly osmotic as it may be reversed or retarded by dilute solutions of sucrose. Rupture of the membrane is apparently spontaneous and dependent on reaching a sufficient degree of stretch. The animals 


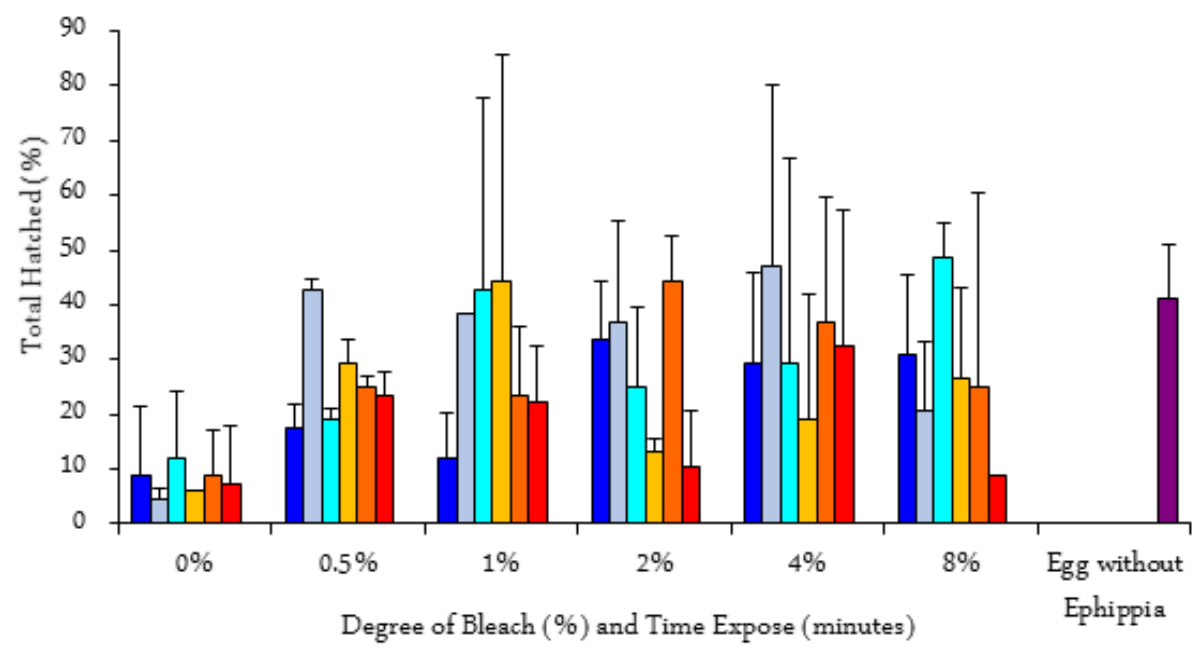

1 minutes $\square^{2}$ minutes $\square 4$ minutes $\square^{8}$ minutes $\square^{16}$ minutes $\square 32$ minutes

Figure 1. Average total cumulative hatch each treatment after 25 days incubation (sample size was 50 ephippia per jar and 2 jars/replicates per treatment combination)

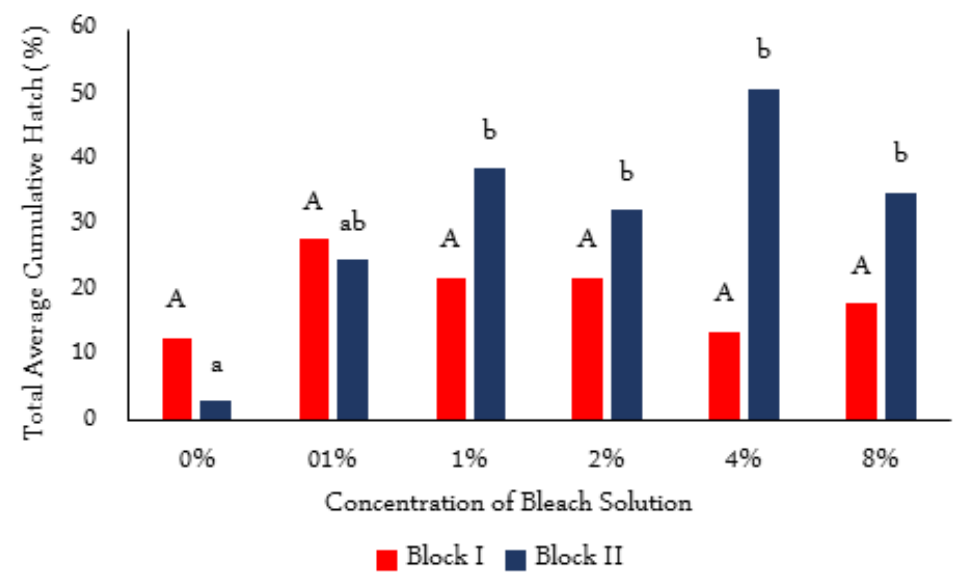

Figure 2. Average total cumulative hatch of D. magna ephippial eggs per treatments concentration of bleach solution within 25 days of incubation (note: the levels in the same block no connected by same letter are significantly different based on Tukey HSD at $\alpha=0.05$ )

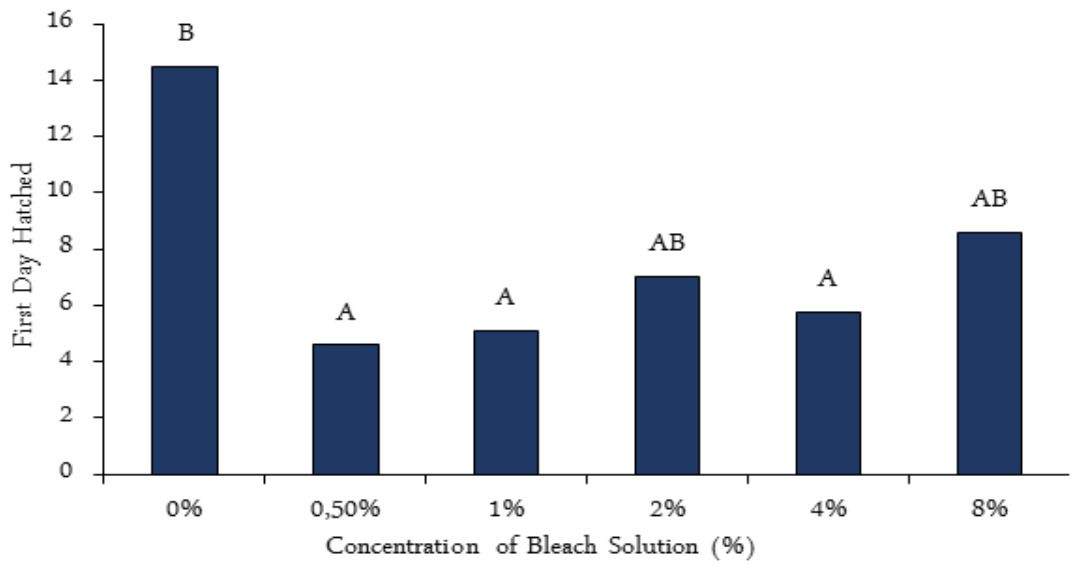

Figure 3. The average time period until the first hatch of $D$. magna ephippial eggs per treatments concentration of bleach solution (note: levels not connected by same letter are significantly different based on Tukey HSD at $\alpha=0.05$ ) 
molt a few minutes after escape, acquiring at this molt the caudal spine characteristic of Daphnia. At $24^{\circ} \mathrm{C}$ and 56 foot candle of light, development is complete 55 hr after decapsulation.

From Figure 1, we can see a trend, that exposing ephippia to bleach solution with a longer time (32 minutes) at high concentration (8\%) and short time (1 minutes) at low concentration $(0.5 \%$ and $1 \%)$ caused decreasing of $D$. magna hatching. High concentration of bleach exposure times up to 16 minutes may not influence the hatching of $D$. magna resting eggs negatively. High toxicity of bleach solution up to $16 \mathrm{~min}$ utes exposing did not break the morphology and anatomy of eggs. It might be because the ephippia preserve eggs from external environment.

Based on full factorial two-way ANOVA (Table 1), there is a significant difference in total cumulative hatching between block $(\mathrm{F}=9.28, \mathrm{P}=0.00)$ and among concentration of sodium hypochlorite $(\mathrm{F}=3.68, \mathrm{P}=$ $0.01)$, but this contrast did not differ among time exposing $(\mathrm{F}=1.30, \mathrm{P}=0.29)$ and no interaction between concentration of $\mathrm{NaClO}$ and time exposing $(\mathrm{F}=0.85$, $\mathrm{P}=0.66)$. The difference between this block indicates that there is a difference in quality of ephippia. It was due to ephippia have been stored at $4^{\circ} \mathrm{C}$ in complete darkness for seven years. Because there is no difference total cumulative hatching between block and treatments of time exposing, so we did one way ANOVA tests between the concentration of $\mathrm{NaClO}$ on each block as shown in Figure 2. From this figure we can see in the block II that treatment with bleach solution will increase the hatching rate by about $21 \%$ over unbleached controls. However these was no significant difference among the 5 levels of bleach treatments.

Using eggs without the ephippia and no treat with bleach solution result $41 \%$ hatching, which is not difffferent from the group of ephippia that received a bleach solution treatment (Figure 1). During the experiment, we observed that treatments with $4 \%-8 \%$ bleach exposing 8 to 32 minutes caused a decapsulationof eggs. The ephippial is not a barrier to an adequate light stimulus but does constitute a powerful bar-

Table 1. Two way ANOVA of total cumulative hatching (\%)

\begin{tabular}{lcc}
\hline \multicolumn{1}{c}{ Source } & F. Ratio & Prob. $>F$ \\
\hline Block & 9.28 & 0.00 \\
Concentration of $\mathrm{NaClO}$ & 3.68 & 0.01 \\
Exposure time & 1.30 & 0.29 \\
Concentration of $\mathrm{NaClO}^{*}$ time exposing & 0.85 & 0.66 \\
\hline
\end{tabular}

rier to the completion of development [17]. If we judge from the tightly sealed, heavy walled character of the ephippium, the inhibition is most probably a direct interference with the diffusion between the egg and the external environment.

Heavy walled character of ephippium preserve eggs from external environment. Based on our results, we suspect that bleach solution breaks the tightly sealed ephippium so that the diffusion process of the eggs with external environment is fascilitated, leading to a more rapid completion of egg development. The effect of $\mathrm{NaClO}$ was its ability to permit an immediate response to light [13]. But [14] found that whatever the effect of hypochlorite in interrupting further diapause development, its effectiveness depended on subsequent exposure to light.

\section{The time period until first hatching}

The average time period until the first hatching in a jar was observed varied from 3.5 to 20.5 days. Based on full factorial two-way ANOVA (Table 2), there is a significant difference of days until first hatch among concentration of $\mathrm{NaClO}(\mathrm{F}=4.24, \mathrm{P}=0.00)$. These were no effect of Block $(\mathrm{F}=0.47, \mathrm{P}=0.50)$, exposure time $(\mathrm{F}=0.31$, $\mathrm{P}=0.90)$ and interaction between concentration of $\mathrm{Na}$ $\mathrm{ClO}$ and exposure time to the first day hatching. Based on the result, so we did one way ANOVA tests between the concentration of $\mathrm{NaClO}$ as shown in Figure 3.

Figure 3 shows that in the treatments with bleach solutions of $0.5 \%, 1 \%$ and $4 \%$ hatching occurred significantly earlier (average hatched on day 4.6-5.8) than concentration $2 \%$ and $8 \%$ (average hatched on day 7.0 and 8.6 respectively). The ephippia without bleach treatment ( $0 \%$ control treatment) needed much longer times to first hatch (average hatched on day 14.2). Generally, $10 \%$ of the total hatch was obtain on the first or second day after hatching and $25 \%$ of the totalhatch was found on the third day after hatching. This experiment indicated that $\mathrm{NaClO}$ is required to induce the hatching of $D$. magna resting eggs in lower concentration. But in the high concentration, bleaching so

Table 2. Two way ANOVA of time period until the first hatching

\begin{tabular}{lcc}
\hline \multicolumn{1}{c}{ Source } & F. Ratio & Prob. $>F$ \\
\hline Block & 0.47 & 0.50 \\
Concentration of $\mathrm{NaClO}$ & 4.24 & 0.00 \\
Exposure time & 0.31 & 0.90 \\
Concentration of $\mathrm{NaClO}^{*}$ time exposing & 0.75 & 0.77 \\
\hline
\end{tabular}




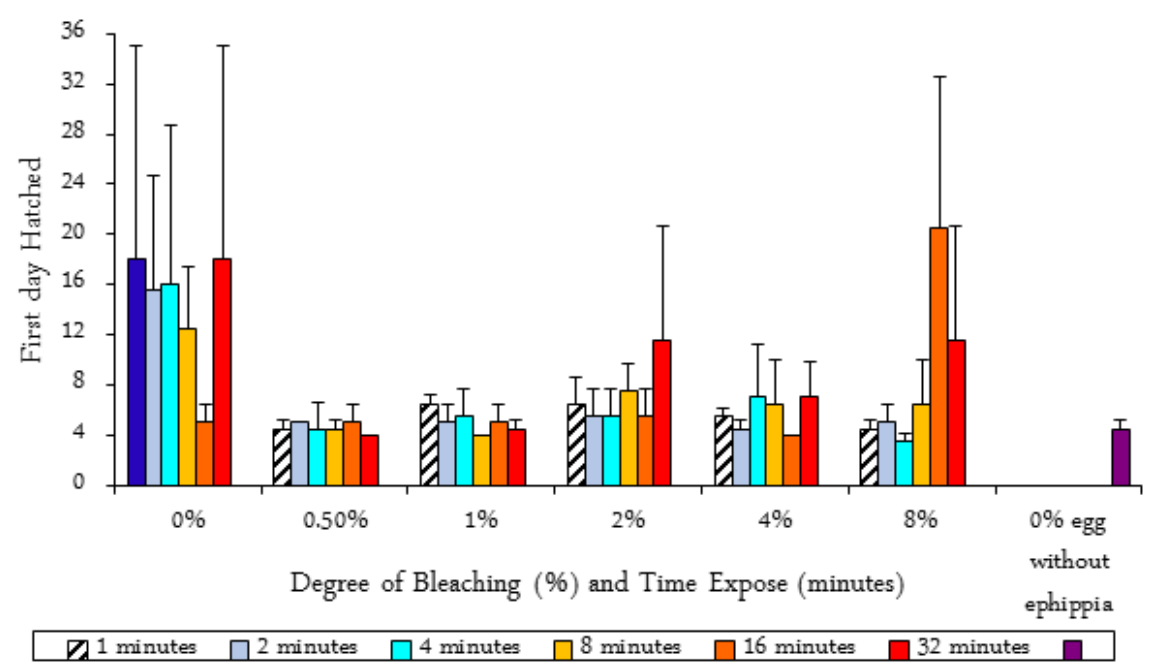

Figure 4. The average of time period until the first hatching of D. magna ephippia eggs per treatments concentration of bleach solution across exposure time

lution will be inhibited the release of diapause due to the toxicity of this substance. Although there was no significant difference the first day of hatch among time expose of bleach solution (Table 2), but from Figure 4 we can see that the longer time exposing (32 minutes) on the higher concentration of bleach solution $(2 \%$, $4 \%$ and $8 \%$ ) needed the longer time to first hatch (711.5 days). It was happened too on the concentration of bleach solution $8 \%$ and time exposed 16 minutes with the average first hatched on day 20.5 days. It was indicated that $\mathrm{NaClO}$ in the high concentration $(2 \%$, $4 \%$ and $8 \%$ ) and longer exposure times (16 and 32 minutes) will be inhibit the development of embryo might be because it is an effective disinfectant agent that has been known having high toxicity.

From the observation of two block, there was found that the range of time minimum needed to first hatched of ephippial eggs generally is 3-6 days, except the control treatment (4-9 days) and the treatment concentration of bleach solution $8 \%$ crossed with exposure time 16 minutes (12 days). Eggs prepared out from ephippia when didn't give a treatment with bleach solution need 4-5 days to first hatched (Figure 4).

The implication of this experiment that although $\mathrm{NaClO}$ can induce the hatching of $D$. magna resting eggs, but in the higher concentration and longer exposure times will be inhibit the development of embryo. $\mathrm{NaClO}$ is an effective disinfectant agent that has been known having high toxicity. It might cause the lethal effect to bacteria and fungi surrounding ephippia and so the hatching success is will be higher. In the water $\mathrm{NaClO}$ will become ion of sodium and chloride. Sodium is one of the most abundant elements and is a common constituent of natural waters. The presence of chlorides in natural waters can mainly be attributed to dissolution of salt deposits in the form of ions (Cl-). It is the major form of inorganic anions in water for aquatic life.

\section{CONCLUSION}

Preliminary treatment with $0.5-8 \% \mathrm{NaClO}$ (bleach solution) significantly increases the yield of total hatch rate of $D$. magna resting eggs by about $21 \%$ over unbleached control. However, there was no significant difference among the bleach treatments. Concentration of bleach solution $0.5 \%, 1 \%$ and $4 \%$ significantly accelerated the time period until the first day hatching. Difference of exposure time (1-32 minutes) at each concentration treatments were not influence the yield of total hatch and the time period until first hatching.

These result showed that $\mathrm{NaClO}$ can induce the hatching of $D$. magna resting eggs which can then be used in research experiment in the field of ecology, genetics, toxicology and evolutionary studies.

\section{ACKNOWLEDGMENT}

I thank to members of the Ecology and Evolution Unit, Biology Department, Fribourg University Switzerland for permitted and support this research and thank you for project of TPSDP 2004, Biology Department, Faculty of Mathematics and Natural Sciences Brawijaya University for giving the fund for this trainer-ship so this research can be done.

\section{REFERENCES}

1. Haag CR, Hottinger JW, Riek M, Ebert D (2002) Strong 
inbreeding depression in a Daphnia metapopulation. Evolution 56 (3): 518-526.

2. Curtis H, Barnes NS (1989) Biology: Fifth Edition. Worth Publishers Inc., New York. New York.

3. Terra NR, Feiden IR (2003) Reproduction and survival of Daphnia magna Straus, 1820 (Crustacea: Cladocera) under different hardness conditions. Acta Limnol. Bras. 15 (2): 51-55.

4. Balcer MD, Korda NL, Dodson SI (1984) Zooplankton of the Great Lakes. University of Wisconsin Press. Madioson.

5. Hobæk A, Larsson P (1990). Sex determination in Daphnia magna. Ecology 71 (6): 2255-2268.

6. Winsor GL, Innes DJ (2002) Sexual reproduction in Daphnia pulex (Crustacea: Cladocera): observations on male mating behaviour and avoidance of inbreading. Freshwater Biology 47: 441-450.

7. Haag CR, Sakwiñska O, Ebert D (2003) Test of synergistic interaction between infection and inbreeding in Daphnia magna. Evolution 57 (4): 777-783.

8. Hebert PDN (1978)The population biology of Daphnia (Crustacea, Daphnidae). Biological Reviews 53: 387-426.

9. Innes DJ (1997) Sexual reproduction of Daphnia pulex in a temporary habitat. Oecologia 111: 53-60.
10. Hutchinson GE (1967) A treatise on limnology Vol II. John Wiley and Sons. New York.

11. Mellors WK (1975) Selective predation of ephippial Daphnia and the resistance of ephippial eggs to digestion. Ecology 56: 974-980.

12. Slusarczyk M (2001) Food threshold for diapause in Daphnia under the threat of fish predation. Ecology 82 (4): 1089-1096.

13. Pancella JR, Stross RG (1963) Light induced hatching of Daphnia resting eggs. Chesapeake Sciences 4 (3): 135-140

14. Stross RG (1966) Light and temperature requirements for diapause development and release in Daphnia. Ecology 47: 368-374.

15. Stollewerk A (2010). The water flea Daphnia - a 'new' model system for ecology and evolution?. Journal of Biology 9: 21.

16. Roulin AC, Routtu J, Hall MD et al. (2013) Local adaptation of sex induction in a facultative sexual crustacean: insight from QTL mapping and natural populations of Daphnia magna. Molecular Ecology 22: 3567-3579.

17. Davison J (1969) Activation of the ephippial egg of Daphnia pulex. The Journal of General Physiology 53: 562-575. 